\title{
RAPID PARAMETRIC HUMAN MODELING IN 3D GARMENT SIMULATION
}

\author{
Jiajia Peng ${ }^{1, *}$, Gaoming Jiang ${ }^{1,2}$, Honglian Cong ${ }^{1,3}$ \\ ${ }^{1}$ Engineering Research Center for Knitting Technology, Ministry of Education, Jiangnan University, Wuxi, China; \\ ${ }^{2}$ International Joint Research Laboratory for Novel Knitting Structural Materials at Jiangnan University, Wuxi, China; \\ ${ }^{3}$ Key Laboratory of Eco-textiles, Ministry of Education, Wuxi, China; \\ ${ }^{*}$ Corresponding Authors:18206180867@163.com;
}

\begin{abstract}
:
To realize $3 D$ garment simulation online and show the wearing effect of different body types, a method for rapid parametric human modeling is proposed in this article. The parameterization consists of two phases. In the first phase, the characteristic parameters of the sample model are extracted according to the different types of feature information. In the second phase, the deformation is realized by combining the axial deformation method and the radial weight. Thus, according to contrasts between the input measurement and the sample sizes, parametric human modeling is realized by deformation of the sample model. In the deformation stage, the axis curve is simplified to the straight-line segment in the axis deformation method, reducing the calculation. Comparative analysis and the results of experiments demonstrate that the better performance can be achieved at a higher speed, and this method realizes real-time parametric human modeling.
\end{abstract}

\section{Keywords:}

Parametric deformation, Human model, Characteristic sizes

\section{Introduction}

With continuous advancement of the virtual network technology, $3 \mathrm{D}$ garment fitting online and clothing customization have been attached with wide popularity. To realize these advanced functions, the parameterization of human models becomes a necessary part in achieving this and showing the wearing effect of different body types. Therefore, it is necessary to establish a calculation model with high calculating efficiency and favorable effects.

Numerous researches have been conducted for modeling human body. According to the modeling method, they can be classified into primitive construction method, sample interpolation method, and model reconstruction method. The primitive construction [1-3] method is relatively complex and requires a large number of calculation. In contrast with primitive construction method, the sample interpolation method has less calculation [4-6], but it still cannot realize real-time simulation. Model reconstruction [7-8] method has the greatest computing efficiency, and thus this article adopts this method to build a 3D human body model.

Some scholars realize human model reconstruction by modifying the existing model with the spatial geometric deformation technology. Huang [9] proposed free-form deformation method to deform the human model whose result depends on the density of control grids. However, this method will cause cracks after deformation and requires further processing. Dong [10] proposed a radial basis function (RBF) to deform the control points on the human model and applied an interpolation function to calculate the displacement of each point to implement the parametric human modeling. In this method, it needs to extract the characteristic sizes and control points of the human body at first. Lee [8] and Sun [11] proposed an axis deformation method to realize the reconstruction of the human model. However, Lee extracts the contour of the photograph as an axis curve but Sun extracts the internal centerline of the human model as an axis curve. Moreover, the method of Sun needs to find the characteristic size of the human body first. The axis deformation technique used for calculating the local activity frame and the mapping relationship of the axis curve is time consuming. On the basis of the analysis of the above researches, the article proposes a simplified axis deformation method to achieve rapid deformation of the human model.

This article implements parametric human modeling in two steps. The first step is to extract the characteristic information of the sample model. First, according to the human body's standard, a cross-sectional profile of the human body is extracted using a variable-step method. Then, the characteristic points of the human body are divided into bifurcation points, extremum points, and blur points. The bifurcation points are defined using a fuzzy function. Finally, the characteristic size is calculated by the characteristic point information.

In the second step, a deformed body model is obtained using the axis deformation method, which transforms the axis curve into a straight line according to the control points of the human body. A mapping relation is proposed between the points on the human body surface and the line segment. Lastly, a radial deformation weight is added for calculating the deformation in horizon direction. According to the sizes obtained in the first step and the input sizes, the parametric human modeling is performed based on the radial weight and the axial deformation. 


\section{Calculation of characteristic sizes}

The extraction of human body characteristic points can be performed in two steps. First, a cross sections parallel with horizontal plane are moved from the top to the bottom at variable step length and intersected with the human model so that intersecting contour lines can be obtained. Second, analyze whether the characteristic points appear according to the nature of different characteristic points.

\subsection{Extraction of cross section of human body}

The characteristic points and characteristic plane of human body are first determined for the purpose of measuring human body sizes. According to the traditional extraction method of characteristic point, the cross section moves at fixed step length. Smaller step length is adopted in order to get accurate location of characteristic points, but this wastes much search time. In order to improve efficiency, larger step length is adopted, but this affects the searching precision. Thus, variable step length is used in this article to search the characteristic points. The proportional relation between the height of all characteristic points and body height can be obtained from GB16160-2008-T and the study of the Clemson University in the United States [12]. The estimated height values of characteristic parts of human body can be obtained according to equation (1).

$$
h_{x}=\lambda_{x} \mathrm{H}
$$

where $H$ refers to the body height, $x$ refers to a characteristic point, and $\lambda_{x}$ refers to the proportionality coefficient of characteristic height to body height.

In order to improve the searching efficiency, only the area of characteristic points should be searched. The search range of the characteristic points adopts the middle height between this characteristic point and last characteristic point as the upper limit and the middle height between this characteristic point and next characteristic point as the lower limit. The step length for searching can be calculated using the following equation:

$$
\varepsilon_{i}=\frac{h_{i}-h_{x}}{h_{x}} \times \frac{H}{500}
$$

However, when $h_{i}=h_{x}$ and step length $\varepsilon_{i}=0$, the entire search process would be stagnant. In order to prevent the occurrence of such an endless loop, the value of $\varepsilon_{i}$ should be judged after each calculation. If $\varepsilon_{i}=0, \varepsilon_{i}$ should be set as a very small value, such as $\varepsilon_{i}=0.0005$. According to the searching method of variable step size, when the distance between the search section $S_{i}$ and characteristic point $X$, namely $h_{i}-h_{x}$, is large, the step length $\varepsilon_{i}$ is also big. Similarly, when the distance between the search section $S_{i}$ and characteristic point $X$ is small, the step length $\varepsilon_{i}$ is also small. The cross section sequence generated by variable step size is shown in Figure 1.

The human body models used in this article are the OBJ file derived from other software, in which the human body model surface is composed of triangle. Therefore, the intersection points between cross section and each side of triangles are the intersection points between cross section and human body model. The intersecting sides should be judged first, and then the intersection points between side and cross section can be obtained. The coordinate of intersection point $P$ is obtained using the following equation:

$P_{3}\left(x_{1}-\left(\frac{y_{1}-y_{0}}{y_{1}-y_{2}}\right) \times\left(x_{1}-x_{2}\right) \quad y_{0} \quad z_{1}-\left(\frac{y_{1}-y_{0}}{y_{1}-y_{2}}\right) \times\left(z_{1}-z_{2}\right)\right)$ where $\left(x_{1}, y_{1}, z_{1}\right)$ and $\left(x_{2}, y_{2}, z_{2}\right)$ are the coordinate values of two endpoints on the side intersected with cross section and $y_{0}$ is the height of cross section.

\subsection{Judgement of characteristic point}

The characteristic sizes extracted in this article include height, shoulder breadth, chest circumference, waistline, hipline, leg length, thigh circumference, hand length, and arm circumference. The characteristic points are divided into three categories according to the required characteristic sizes. Category I refers to the bifurcation points, such as armpit point and perineum point. Category II refers to the extreme points, such as chest circumference, waistline, and hipline. Category III refers to fuzzy characteristic information, such as shoulder point and thigh.

The characteristic points of category I are bifurcation points, the intersecting contour lines of which produce the minimum value of intersection angle. The information on characteristic points of the human body is not absolute. Therefore, judging criteria of characteristic should be carried out with uncertain information reasoning ability. A fuzzy rule of membership function of Cauchy distribution has been proposed in this article to identify characteristic points. In the fuzzy theory, the fuzzy set $M$ is depicted by its membership function $\mu_{M}(f)$ that refers to the membership of element $f$ in the fuzzy set M. $\mu_{M}(f)$ makes each object in the fuzzy set $M$ corresponds to a real number within the range $[0,1]$. The value of $\mu_{M}(f)$ closer to 1 leads to greater probability of $f$ being subordinated to $\mathrm{M}$.

$\mathrm{X}_{i}=\left\{P_{i k}, i, k \in N\right\}$ is the intersection point set between search section $S_{i}$ and human model, where $P_{i k}$ is the $k$ th point

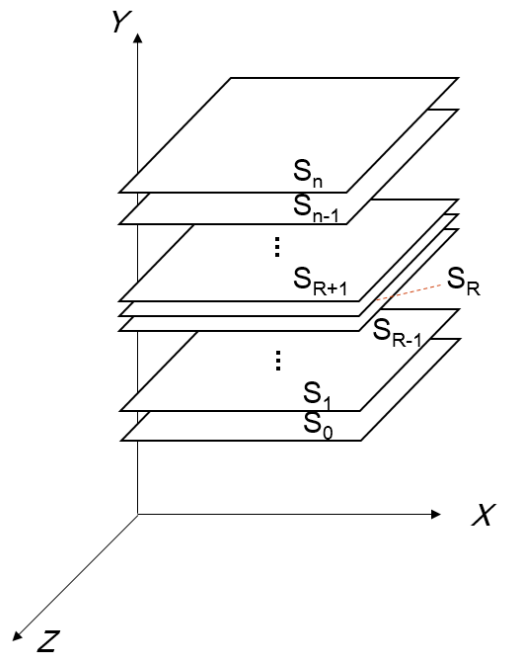

Figure 1. Cross section of sequence 
on the intersecting contour line $X_{i}$. An intersecting contour point set is shown in Figure 2,, where $P_{i k-1}$ is the point on the intersecting contour line $\mathrm{X}_{i}$ next to $P_{i k}$ on the left and $P_{i k+1}$ is the point on the intersecting contour line $X_{i}$ next to $P_{i k}$ on the right. The intersection angle of $P_{i k}$ is defined as $\alpha$, and $\alpha\left(P_{i k}\right)$ can be obtained through cosine law.

$$
\alpha\left(P_{i k}\right)=\arccos \left[\frac{\left(P_{i k} P_{i k-1}\right)^{2}+\left(P_{i k} P_{i k+1}\right)^{2}-\left(P_{i k-1} P_{i k+1}\right)^{2}}{2 \times\left(P_{i k} P_{i k-1}\right) \times\left(P_{i k} P_{i k+1}\right)}\right]
$$

The bifurcation position of human body can be judged from the inflection points of section point set, including perineum points and armpit points. Perineum point is the bifurcation point between thigh and torso. The cross section of perineum point is shown in Figure 2. It can be seen that the cross section with two inflection point is where the perineum point locates. Similarly, there are two inflection points on the characteristic plane of left and right armpit points. Therefore, there are four inflection points on the characteristic plane of armpit points.

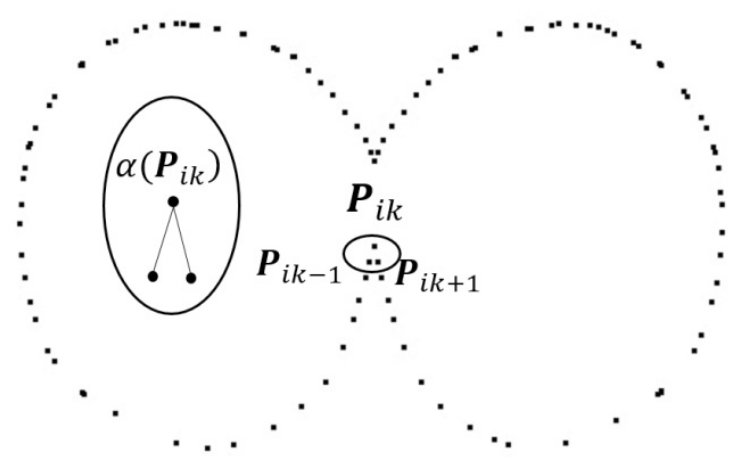

Figure 2. Contour point set and its angle diagram

The fuzzy judgment criterion of inflection points is that if the intersection angle at the inflection point is small enough, the judgment rules of characteristic points of category I are described by taking perineum point as an example.

The angle space can be divided into three subsets, where small angle set is $S=$ Angle Small, general angle set is $M=$ Angle Mid, and large angle set is $L=$ AngleLarge. The membership function of $S$ is $\mu_{\text {AngleSmall }}(\alpha), M$ is $\mu_{\text {AngleMid }}(\alpha)$, and $L$ is $\mu_{\text {AngleLarge }}(\alpha)$. Membership function formulas of set $S, M$, and $L$ are written as follows:

$$
\begin{gathered}
\mu_{\text {AngleSmall }}=\left\{\begin{array}{l}
1, x \leq \text { AngleMin } \\
\frac{1}{1+\frac{(\alpha-\text { AngleMin })^{4}}{\text { AngleMin }}}, x>\text { AngleMin }
\end{array}\right. \\
\mu_{\text {AngleLarge }}=\left\{\begin{array}{c}
0, x \leq \text { AngleMin } \\
\frac{1}{1+\frac{(\alpha-\text { AngleMin })^{-4}}{\text { AngleMin }}}, x>\text { AngleMin }
\end{array}\right. \\
\mu_{\text {Anglemid }}=\frac{1}{1+\frac{\left(\alpha-\text { AngleMin }^{4}\right.}{\text { AngleMin }}}
\end{gathered}
$$

where AngleMin $=\min \left\{\alpha\left(P_{i k}\right), P_{i k} \in \mathrm{X}_{i}\right\}$ and the membership of $\alpha\left(P_{i k}\right)$ in three sets can be compared to determine which set $\alpha\left(P_{i k}\right)$ is subordinated to.
According to the computed results through formula (5), (6), and (7), respectively, the point $P_{i k}$ on the intersecting contour line $\mathrm{X}_{i}$ belongs to the degree of membership $\mu_{\text {Small }}\left(\alpha\left(P_{i k}\right)\right)$, $\mu_{\text {Mid }}\left(\alpha\left(P_{i k}\right)\right)$, and $\mu_{\text {Large }}\left(\alpha\left(P_{i k}\right)\right)$ of $S, M$, and $L$. The fuzzy judgment rules of the inflection points are made as follows:

$$
\begin{aligned}
& \text { i. If }\left(\mu_{\text {Small }}\left(\alpha\left(P_{i k}\right)\right)>\mu_{\text {Mid }}\left(\alpha\left(P_{i k}\right)\right)\right) \& \&\left(\mu_{\text {Small }}\left(\alpha\left(P_{i k}\right)\right)>\mu_{\text {Large }}\left(\alpha\left(P_{i k}\right)\right)\right), \\
& \alpha\left(P_{i k}\right) \in \text { Small . }
\end{aligned}
$$

ii. If $\alpha\left(P_{i k}\right) \in$ Small , $P_{i k}$ is the inflection point.

The perimeters of characteristic points of category II are compared to determine the position of characteristic points, where $X_{j}$ is the intersection point set between the ith search section $S_{i}$ and human body and $I_{i}$ is the length of section profile calculated from the intersection point set. The cross section of the characteristic points is from $S_{i}$ to $S_{m}$. The sets of intersection points between the cross section of the characteristic point and human body are from $X_{i}$ to $X_{m}$. The length of cross section is from $I_{i}$ to $I_{m}$. $L_{t}$ is the set of length of all cross section. To take the chest circumference point as an example, the judgment rule of the characteristic points appear is written as follows:

$$
I_{\text {max }}=\max \left\{L_{t}, t \in N\right\}, \text { where } L_{t}=\left\{I_{i}, I_{i+1}, \ldots, I_{m-1}, I_{m}\right\}
$$

The cross section of $I_{\text {max }}$ is the characteristic cross section of chest circumference. The characteristic points of category II include chest circumference, waistline, and hipline. The cross section of chest circumference and hipline have the longest sections profile, and the cross section of waistline has the shortest section profile.

The points of category III are used for shoulder breadth and thigh circumference. The height of characteristic points of category III can be calculated from the proportional relation between the height of all characteristic points of human body and body height.

\subsection{Calculation of characteristic sizes}

The characteristic sizes of human body are divided into two categories in this article. The first category refers to vertical linear size, such as body height and perineum height. The second category refers to level circumference size, such as chest circumference, waistline, and hipline. $Y$ coordinate difference value method is used in this article to calculate the characteristic sizes of the first category. The value of points in the OBJ file derived from the software is not the actual value of human body and there exists some proportional relation, so the body height of model is set as $\mathrm{H}$. The height of characteristic cross section is calculated as follows:

$$
h_{t}=\left(y_{t}-y_{\min }\right) \times\left(y_{\max }-y_{\min }\right) / H
$$

where $y_{t}$ refers to the coordinate of characteristic cross section on $\mathrm{Y}$-axis and $y_{\min }$ refers to the minimum coordinate value of point cloud data of human body on Y-axis. 
The perimeters of intersecting profiles are obtained to calculate the characteristic sizes of the second category.

The point at the left bottom obtained from the search point set $\mathrm{X}_{t}$ is $P_{1}$ and the unit vectors that $P_{1}$ points to other points in point set are obtained. The unit vector of the second point has the largest height value, and $P_{2}$ is the second point as shown in Figure 3(a). The vector $\vec{P}_{2} P_{1}$ is obtained from the point $P_{1}$ and $P_{2}$. Then the cosine values are obtained between $\overrightarrow{P_{2} P_{1}}$ and the unit vectors $P_{2}$ pointing to other points in point set. And the cosine value of the third point is largest. This method can be used to obtain other contour points required. If the point obtained is $P_{1}$, search should be stopped, and the obtained point set that forms a convex polygon is shown in Figure 3(b). The circumference corresponding to characteristic points is obtained according to formula (10).

$$
I_{x}=\sum_{k=1}^{n} I\left(P_{k}, P_{k+1}\right)+I\left(P_{n}, P_{1}\right)
$$

where the function $I\left(P_{k}, P_{k+1}\right)$ is the distance between the point $P$ and the point $P_{k+1} ; I\left(P_{n}, P_{1}\right)$ is the distance between the last point and the first point.

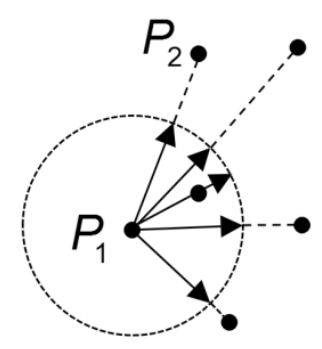

(a)

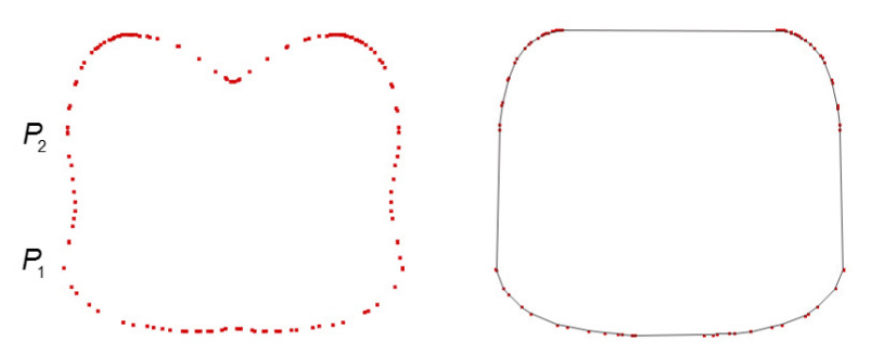

(b)

Figure 3. Convex polygon of cross section

\section{Parametric deformation of human body}

According to the definition of characteristic points of human body, 19 main control points are established on the standard model of human body. To take the standard female model as a simple, the diagrammatic drawing of control points on the model is shown in Figure 4. Three-dimensional axial deformation technology is used in this article to achieve parameterized human body deformation, and human body models in different sizes can be generated from the deformation of template model in accordance with the control points. The template model is divided into six parts when it is parameterized, namely, head, body, left arm, right arm, left leg, and right leg. The length and circumference of all parts of human body should be adjusted automatically according to the variation of parameter values of morphological characteristics, so as to realize the parametric deformation of model of human body.

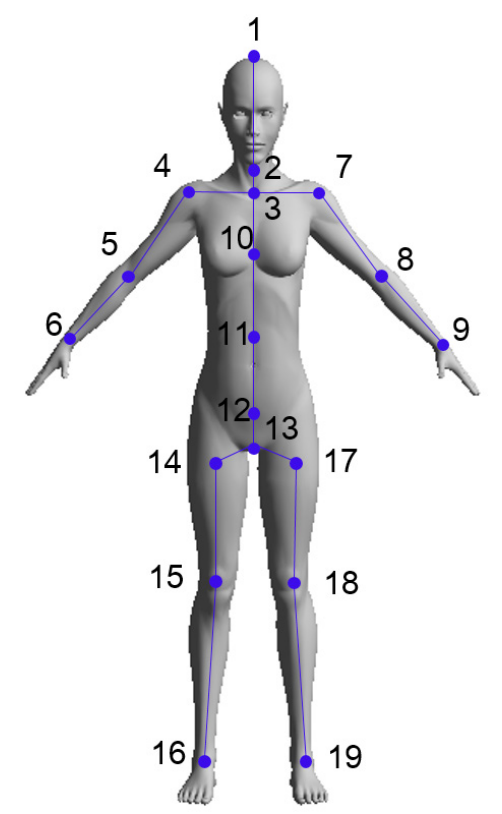

Figure 4. Control points on the human model

\subsection{Axial deformation algorithm}

The shape of template model can be changed through axis by three-dimensional axis [13]. First, axis curves should be generated for each part of human body in order to realize axial deformation of human body model. Second, each point of human body surface is mapped to the corresponding axis curves. And then the axis curves are adjusted to get the corresponding coordinates after axial deformation. Finally, inverse transformation of the original mapping relation should act on the human body surface to achieve deformation. The axis curves have been simplified to be straight lines for the purpose of simplifying calculation in this article. The following example of arm explains the arm deformation achieved by three-dimensional axial deformation method. As shown in Figure 5 , the three-dimensional axis $L$ on the upper arm is a straight-line segment connected by the control point 7 and 8 (point $P_{1}$ and $P_{2}$ in the figure). Point $A$ is a vertex on human body surface of upper arm, and point $B$ is the point mapped by the point $A$ to the axis $L$.

The three-dimensional coordinate of point $A$ on human body, surface, is $\left(x_{A}, y_{A}, Z_{A}\right)$, the coordinate of $P_{1}$ is $P_{2}^{\prime}\left(x_{P 2}^{\prime}, y_{P 2}^{\prime}, z_{P 2}^{\prime}\right)$, and the coordinate of $P_{2}$ is $\left(x_{P 2}, y_{P 2}, z_{P 2}\right)$. So the mathematical expression of the axis $L$ is written as follows:

$$
\frac{x-x_{p 1}}{x_{p 2}-x_{p 1}}=\frac{y-y_{p 1}}{y_{p 2}-y_{p 1}}=\frac{z-z_{p 1}}{z_{p 2}-z_{p 1}}
$$

Point $B$ is the projection point from point $A$ to the axis $L$, that is, the value of cross product of the vector $\overrightarrow{A B}$ and axial vector $P_{1} P_{2}$ is 0 . The expression is written as follows:

$\overrightarrow{A B} \times \overrightarrow{P_{1} P_{2}}=\left(x_{B}-x_{A}\right)\left(x_{p 2}-x_{p 1}\right)+\left(y_{B}-y_{A}\right)\left(y_{p 2}-y_{p 1}\right)+\left(z_{B}-z_{A}\right)\left(z_{p 2}-z_{p 1}\right)=0$ 
Because the mapping point $B$ of point $A$ on the axis is on the line segment $P_{1} P_{2}$, the value of $k$ can be obtained from formulae (13) and (14).

$$
\begin{gathered}
k=\frac{x_{B}-x_{p 1}}{x_{p 2}-x_{p 1}}=\frac{y_{B}-y_{p 1}}{y_{p 2}-y_{p 1}}=\frac{z_{B}-z_{p 1}}{z_{p 2}-z_{p 1}} \\
k=\frac{\left(x_{p 2}-x_{p 1}\right)\left(x_{A}-x_{p 1}\right)+\left(y_{p 2}-y_{p 1}\right)\left(y_{A}-y_{p 1}\right)+\left(z_{p 2}-z_{p 1}\right)\left(z_{A}-z_{p 1}\right)}{\left(x_{p 2}-x_{p 1}\right)^{2}+\left(y_{p 2}-y_{p 1}\right)^{2}+\left(z_{p 2}-z_{p 1}\right)^{2}}
\end{gathered}
$$

The three-dimensional coordinate of $B$ can be obtained as follows:

$$
B:\left(k \times\left(x_{p 2}-x_{p 1}\right)+x_{p 1}, \quad k \times\left(y_{p 2}-y_{p 1}\right)+y_{p 1} \quad k \times\left(z_{p 2}-z_{p 1}\right)+z_{p 1}\right)
$$

In the axis transformation of axial deformation calculation, the positions of control points are changed according to the input sizes, and the coordinates of $P_{1}$ and $P_{2}$ are changed to be $P_{1}^{\prime}\left(x_{P 1}^{\prime}, y_{P 1}^{\prime}, z_{P 1}^{\prime}\right)$ and $P_{2}^{\prime}\left(x_{P 2}^{\prime}, y_{P 2}^{\prime}, z_{P 2}\right)$. The axis $L$ is a straight-line segment, and the proportional relation between the mapping points and two control points remains unchanged before and after the change, so the value of $k$ can be expressed as follows:

$$
k=\frac{x_{B}-x_{p 1}}{x_{p 2}-x_{p 1}}=\frac{x_{B}^{\prime}-x_{p 1}^{\prime}}{x_{p 2}^{\prime}-x_{p 1}^{\prime}}
$$

The coordinate of the mapping point $\mathrm{B}^{\prime}$ after the change is obtained as follows:

$$
B^{\prime}:\left(\frac{k \times y_{p 2}^{\prime}+y_{p 1}^{\prime}}{1+k}, \frac{k \times y_{p 2}^{\prime}+y_{p 1}^{\prime}}{1+k} \frac{k \times z_{p 2}^{\prime}+z_{p 1}^{\prime}}{1+k}\right)
$$

The distance between the two points after the change remains unchanged, so the coordinate of the point $A^{\prime}$ after the change is obtained as follows:

$$
A^{\prime}:\left(x_{A}+\left(x_{B}^{\prime}-x_{B}\right), \quad y_{A}+\left(y_{B}^{\prime}-y_{B}\right) \quad z_{A}+\left(z_{B}^{\prime}-z_{B}\right)\right)
$$

\subsection{Radial deformation}

The axial deformation method based on the control point provides the mapping relation between point and axis. And it solves the deformation of human body model in the lengthwise direction. The deformation perpendicular to the axis is called as radial deformation in this article. Specifically, it takes the area between the waistline and chest circumference as an example.

As shown in Figure 6 the control point of chest circumference is $P_{3}$, the control point of waistline is $P_{4}, C\left(x_{C}, y_{C}, z_{C}\right)$ is a vertex of human body surface in the interval, and point $D$ is the mapping point of the vertex $C$ mapped to the axis. If the specific value of chest circumference between the user and the standard model is $r_{1}$, the proportion of waistline between the user and the standard model is $r_{2}$. For the purpose of smooth transition of different areas of human body model, the radial deformation in the interval between the waistline and chest circumference needs to be calculated according to $r_{1}$ and $r_{2}$. The computing method of radial deformation weight is given in formula (19).

$$
w=\left(r_{1}-1\right) / r_{1} \times \frac{D P_{3}}{P_{3} P_{4}}+\left(r_{2}-1\right) / r_{2} \times \frac{D P_{4}}{P_{3} P_{4}}
$$

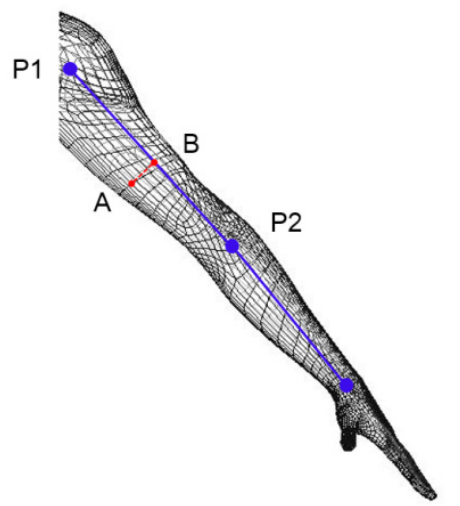

Figure 5. The three-dimensional axis on the upper arm

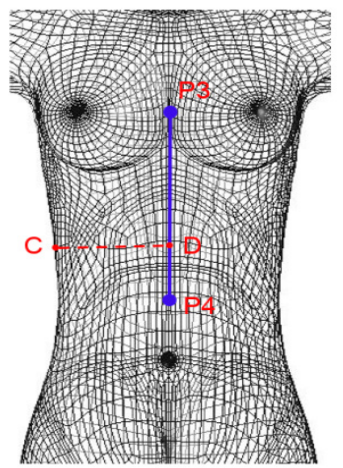

Figure 6. Schematic diagram of radial deformation

where $W$ is the radial weight, $D P_{3}$ is the distance from point $D$ to point $P_{3}, D P_{4}$ is distance from point $D$ to point $P_{4}$, and $P_{3} P_{4}$ is the distance from point $P_{3}$ to point $P_{4}$. The mapping point after the change of axis is $D$, the coordinate of point $C$ is $C^{\prime}$, and the coordinate of $C$ is obtained in combination with axial transformation and radial deformation weight, as given in formula (20).

$C^{\prime}:\left(\left(x_{C}+\left(x_{D}^{\prime}-x_{D}\right)\right) \times w,\left(y_{C}+\left(y_{D}^{\prime}-y_{D}\right)\right) \times w,\left(z_{C}+\left(z_{D}^{\prime}-z_{D}\right)\right) \times w\right)$

\section{Results and discussion}

The combined programming of C\# and OPENGL is used to realize characteristic information extraction of human body and parametric deformation through the program flow shown in Figure 7.

\subsection{Characteristic sizes of human body}

In this article, the characteristic sizes were extracted for models with different body types and positions. There are three different simple models, which are shown in Figure 8. Model $X$ is the relative standard body, model $Y$ is shorter and fatter, and model $Z$ is tall and thin. The characteristic sizes of models extracted by the method proposed in this article are shown in Table 1. It can be observed from the table that the waistline and hipline of model $Y$ are larger than those of model $X$, the waistline and hipline of model $Z$ are smaller than those of model $X$, and the chest circumference of model $Y$ and model $Z$ is smaller than that of model $X$. Moreover, Table 1 also reflects other informations, such as the height and leg length of model 

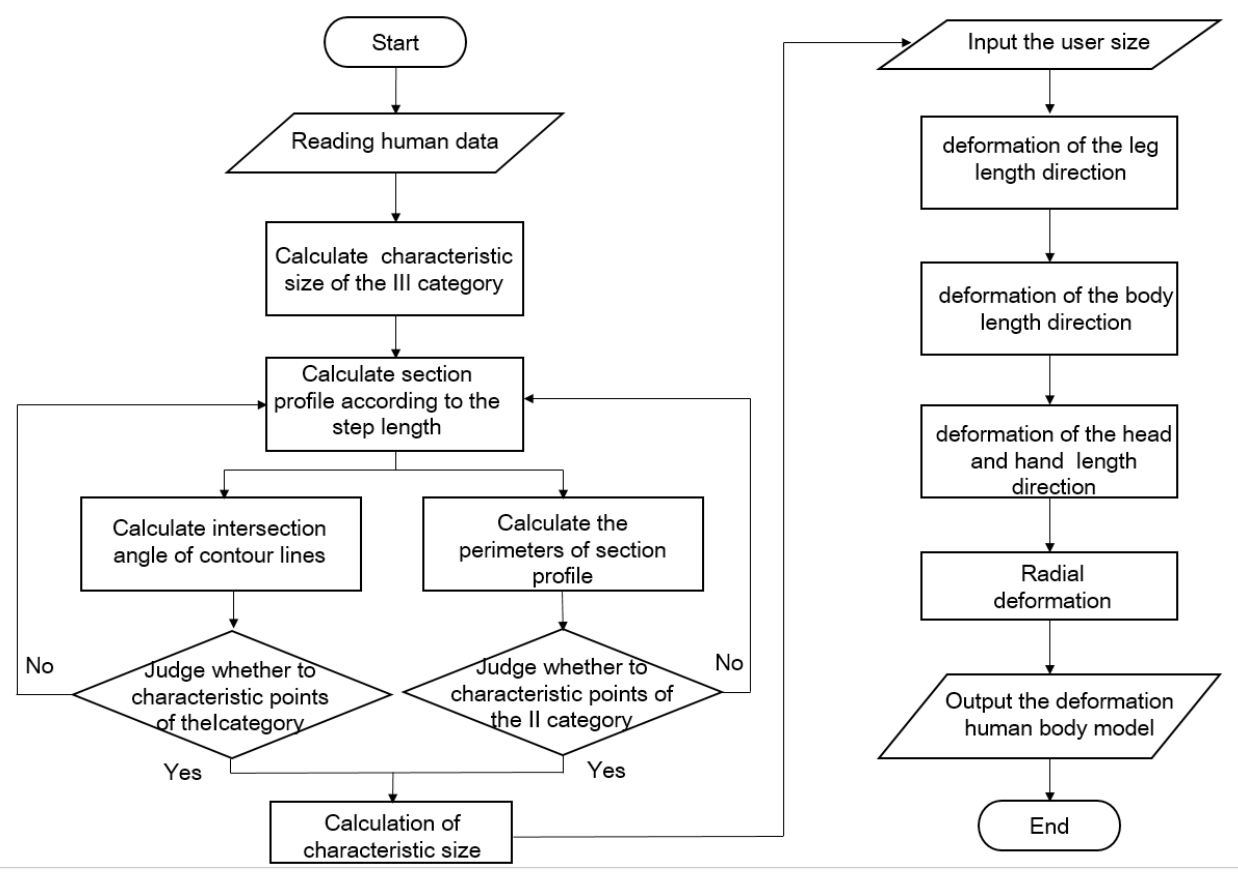

Figure 7. Work flow diagram

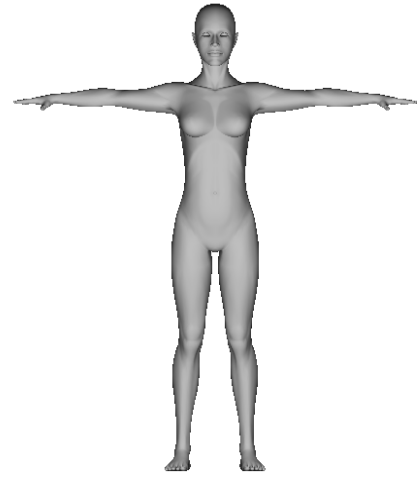

Model X

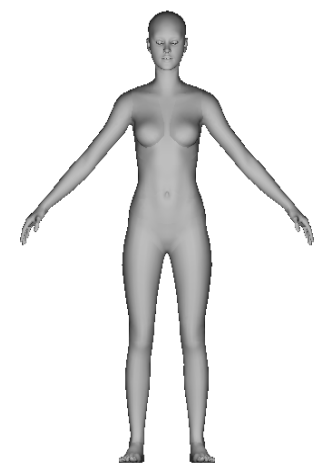

Model Y

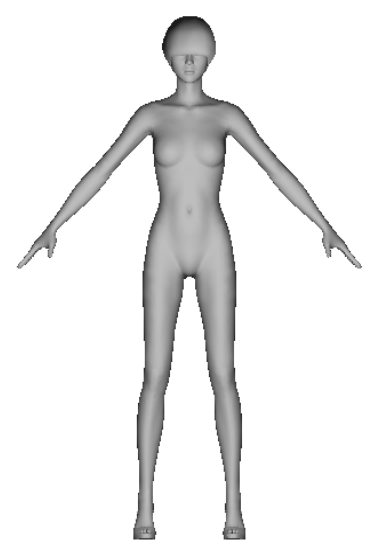

Model Z

Figure 8. Template body models

Table 1. The extraction results of important characteristic sizes of sample human body (unit: $\mathrm{mm}$ )

\begin{tabular}{|l|c|c|c|}
\hline Sizes & Model X & Model Y & Model Z \\
\hline Body height & 165 & 160 & 373 \\
\hline Neck circumference & 35 & 32 & 38 \\
\hline Shoulder breadth & 36 & 35 & 85 \\
\hline Chest circumference & 94 & 85 & 58 \\
\hline Waistline & 62 & 68 & 91 \\
\hline Hipline & 96 & 104 & 79 \\
\hline Leg length & 75 & 74 & 48 \\
\hline Thigh circumference & 51 & 51 & 62 \\
\hline Hand length & 58 & 62 & 22 \\
\hline Arm circumference & 22 & 24 & 32 \\
\hline
\end{tabular}


$Z$ are larger than those of model $X$ and model $Y$, but the thigh circumference is smaller. From the above analysis, it can be concluded that the extracted characteristic sizes correctly reflect the model type in Figure 8. Therefore, it proves that the method extracted is more accurate.

\subsection{Parametric deformation}

Figure 9 shows the models of human body deformation in different measurements, in which the models are obtained from the template model deformation based on the specified measurements. In Figure 9, the sample model is the model $X$ in Figure 8, and the model $A$ to model $D$ are the human body with deformation. The specific measurements of model $X$ and model $A$ to model $D$ are given in Table 2. In addition, model $A$ has undergone local deformation based on the original sample model, and model B to model D have undergone overall deformation. It can be concluded from the results that the parameterized deformation method proposed in this article can realize the local deformation and the overall deformation, and the results of the human body model are fine.

\subsection{Analysis of efficiency}

In the reference mentioned, references [10] and [11] extracted characteristic information before deformation and then reconstructed it according to the input measurements, which are similar to this article. Therefore, this article compares the efficiency of the two methods.

The time consumed by the modeling of reference [10] is compared with the time consumed in this article. Table 3 shows the time consumed to implement the modeling in Figure 9, which use the method of this article and the method adopted in reference [10]. From the data given in the table, it can be concluded that the method proposed in this article is nearly one order of magnitude faster than the method proposed in reference [10]. In reference [10], RBF and interpolation algorithms were used to implement model reconstruction and

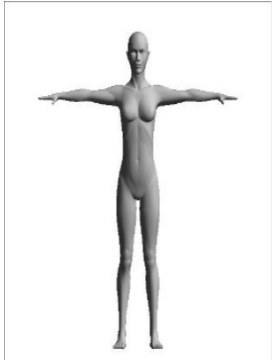

Standard model

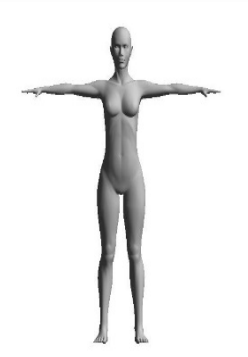

Model A

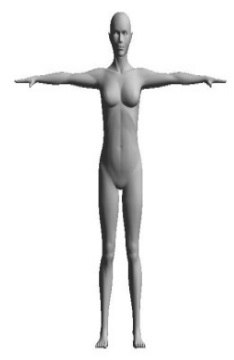

Model B

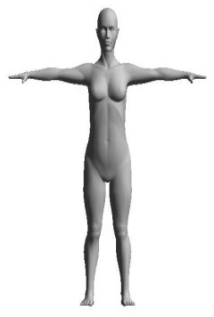

Model C

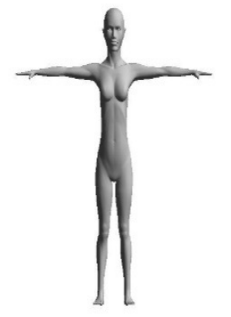

Model D

Figure 9. New human body model generated from the template body model deformation based on the sizes in Table 2

Table 2. Different user-specific sizes (unit: $\mathrm{mm}$ )

\begin{tabular}{|c|c|c|c|c|c|}
\hline Sizes & $\begin{array}{c}\text { Standard } \\
\text { model }\end{array}$ & Model A & Model B & Model C & Model D \\
\hline Body height & 165 & 165 & 175 & 155 & 155 \\
\hline Neck circumference & 35 & 35 & 35 & 35 & 35 \\
\hline Shoulder breadth & 36 & 36 & 36 & 36 & 34 \\
\hline Chest circumference & 94 & 94 & 100 & 100 & 78 \\
\hline Waistline & 62 & 66 & 70 & 80 & 55 \\
\hline Hipline & 96 & 110 & 95 & 106 & 85 \\
\hline Leg length & 75 & 75 & 80 & 62 & 62 \\
\hline Thigh circumference & 51 & 55 & 48 & 55 & 42 \\
\hline Hand length & 58 & 59 & 61 & 58 & 58 \\
\hline Arm circumference & 22 & 20 & 22 & 23 & 19 \\
\hline
\end{tabular}

Table 3. Modeling time comparison (unit: $\mathrm{ms}$ )

\begin{tabular}{|c|c|c|c|c|c|c|}
\hline Method & $\begin{array}{c}\text { Number of } \\
\text { Vertexes }\end{array}$ & $\begin{array}{c}\text { Number of } \\
\text { face }\end{array}$ & Model A & Model B & Model C & Model D \\
\hline $\begin{array}{c}\text { Proposed } \\
\text { Method }\end{array}$ & 58,591 & 115,520 & 4 & 5 & 4 \\
\hline RBF & 58,591 & 115,520 & 38 & 41 & 37 & 38 \\
\hline
\end{tabular}


the interpolation calculation for each vertex consumed more time.

Because there is no related data for modeling time and the modeling process used in this article is similar to that in reference [11], this article analyzes efficiency by comparing the methods used in the implementation process. In reference [11], the human body was also deformed by axial deformation, in which the axis curves in the axis deformation were cubic spline interpolation curves. The following is a brief introduction to the parametric modeling process and the methods used in reference [11]. The first step is to calculate the spline interpolation curve function. The second step is to establish the local activity frames of the spline curve. The third step is to establish the relationship between the body surface vertex and the frames. In this step, the interval of the active frame that the vertex belongs to is found and the corresponding local coordinate vector is calculated. The fourth step is to calculate the axis curve required for the deformation and its local moving frames according to the input size. And then the mapping coordinates in the third step are used to calculate the coordinates of the deformed vertex. The method for establishing a local activity frame is to represent the activity frame $F$ as a four-tuple ( $t, T, N, B$ ), where $t$ is the interpolation parameter of the axis corresponding to the spline interpolation curve. The vectors $T, N$, and $B$ are orthogonal to each other and form the three coordinate axes of the activity frame $\mathrm{F}$.

In the method proposed in this article, the first step is to reduce the cubic spline interpolation curve to a straight line. The establishment of the curve local activity framework in the second step is omitted in this article. In the third step, we use the projection of the point on the line as the mapping relationship, which reduces the calculation compared to the method used in reference [11]. The fourth step does not recalculate the local motion frame of the new curve. Therefore, it can be concluded that the proposed method is simpler than the one used in reference [11]. In other words, the calculation time is less.

From the above analysis, it can be concluded that the proposed method is faster and can achieve real-time human modeling.

\section{Conclusion}

For the timeliness of parametric human body modeling, space geometry deformation is used in this article to realize the rapid generation of human body. The method of changing step length is adopted to search the characteristic points in accordance with the relevant standard of human body so as to reduce calculated amount. The membership function of Cauchy distribution and fuzzy rule are used to judge the characteristic points, and the results are accurate. The axis curves are simplified as straight line for axial deformation, and the radial deformation weight is increased at the same time, which realizes the timeliness of deformation and ensure the smoothness of human body model. The combined programming of $\mathrm{C \#}$ and OpenGL is also adopted to realize real-time parametric human body modeling. Finally, the higher efficiency of the method proposed in this article is verified.

\section{Acknowledgments}

The authors acknowledge the financial support from Standard and mode of intelligent manufacturing, Ministry of Industry and Information Technology of the People's Republic of China (No. [2016] 213), Innovation fund project of CIUI (Cooperation among Industries, Universities \& Research Institutes) Jiangsu Province (BY2016022-09) and A Project Funded by the Priority Academic Program Development of Jiangsu Higher Education Institutions (No. [2014]37).

\section{References}

[1] Komatsu K; Human skin model capable of natural shape variation; Visual Computer. 1988,3(5):265-71.

[2] Turner R; Thalmann D; The Elastic Surface Layer Model for Animated Character Construction: Springer Japan; 1993, 399-412.

[3] Thalmann D; Shen J; Chauvineau E; Fast Realistic Human Body Deformations for Animation and VR Applications; Conference on Computer Graphics International 1996.

[4. Allen B; Curless B; Popović Z. The Space of Human Body Shapes: Reconstruction And Parameterization from Range Scans; Acm Transactions on Graphics 2003,22(3):587-94.

[5] Seo H; Magnenat-Thalmann N; An automatic modeling of human bodies from sizing parameters; Symposium on Interactive 3d Graphics 2003.

[6] Seo $H$; Magnenat-Thalmann N;An example-based approach to human body manipulation. Graphical Models 2004,66(1):1-23.

[7] Hilton A; Beresford D; Gentils T; Smith R; Sun W; Virtual People: Capturing Human Models to Populate Virtual Worlds. Computer Animation, 1999 Proceedings 1999.

[8] Lee WS;Gu J; Magnenatthalmann N; Generating Animatable $3 D$ Virtual Humans from Photographs; Computer Graphics Forum 2000.

[9] Huang J; The human modeling process and implementation based on the features points; Southwest Jiaotong University 2007.

[10] Dong Z; Jiang G; Wu Z; Cong H; 3D parametric human modeling for warp-knitted seamless garment. International Journal of Clothing Science \& Technology 2015,27(4):53248.

[11] Sun S; Xu A; Study on parameterized mannequin modeling in 3-D garment simulation. Journal of Textile Research 2007,28(12):89-93.

[12] Pargas R; Automating Information Extraction From 3D Scan Data. Automating Information Extraction Fromd Scan Data 1998.

[13] Lazarus F; Coquillart S; Jancène P. Axial deformations: an intuitive deformation technique. Computer-Aided Design 1994,26(8):607-13. 